IN PREPARATION FOR ApJ. DRAFT OF OCTOBER 6, 2016.

Preprint typeset using LATEX style AASTeX6 v. 1.0

\title{
ON CORRELATED-NOISE ANALYSES APPLIED TO EXOPLANET LIGHT CURVES
}

\author{
Patricio Cubillos ${ }^{1,2}$, Joseph Harrington ${ }^{1}$, Thomas J. Loredo ${ }^{3}$, Nate B. Lust $^{1,4}$, Jasmina Blecic ${ }^{1,5}$, And Madison Stemm ${ }^{1}$ \\ ${ }^{1}$ Planetary Sciences Group, Department of Physics, University of Central Florida, Orlando, FL 32816-2385 \\ ${ }^{2}$ Space Research Institute, Austrian Academy of Sciences, Schmiedlstrasse 6, A-8042 Graz, Austria \\ ${ }^{3}$ Cornell Center for Astrophysics and Planetary Sciences, Space Sciences Building, Cornell University, Ithaca, NY 14853-6801 \\ ${ }^{4}$ Department of Astrophysical Sciences, Princeton University, Princeton, NJ 08544 \\ ${ }^{5}$ Department of Physics, New York University Abu Dhabi, PO Box 129188 Abu Dhabi, UAE
}

\begin{abstract}
Time-correlated noise is a significant source of uncertainty when modeling exoplanet light-curve data. A correct assessment of correlated noise is fundamental to determine the true statistical significance of our findings. Here we review three of the most widely used correlated-noise estimators in the exoplanet field, the time-averaging, residual-permutation, and wavelet-likelihood methods. We argue that the residual-permutation method is unsound in estimating the uncertainty of parameter estimates. We thus recommend to refrain from this method altogether. We characterize the behavior of the time averaging's rms-vs.-bin-size curves at bin sizes similar to the total observation duration, which may lead to underestimated uncertainties. For the waveletlikelihood method, we note errors in the published equations and provide a list of corrections. We further assess the performance of these techniques by injecting and retrieving eclipse signals into synthetic and real Spitzer light curves, analyzing the results in terms of the relative-accuracy and coverage-fraction statistics. Both the time-averaging and wavelet-likelihood methods significantly improve the estimate of the eclipse depth over a white-noise analysis (a Markov-chain Monte Carlo exploration assuming uncorrelated noise). However, the corrections are not perfect, when retrieving the eclipse depth from Spitzer datasets, these methods covered the true (injected) depth within the $68 \%$ credible region in only $\sim 45-65 \%$ of the trials. Lastly, we present our open-source model-fitting tool, Multi-Core Markov-Chain Monte Carlo $\left(\mathrm{MC}^{3}\right)$. This package uses Bayesian statistics to estimate the best-fitting values and the credible regions for the parameters for a (user-provided) model. $\mathrm{MC}^{3}$ is a Python/C code, available at https://github.com/pcubillos/MCcubed.
\end{abstract}

Keywords: methods: statistical — planets and satellites: fundamental parameters — techniques: photometric

\section{INTRODUCTION}

Whether one's goal is the detection or the characterization of exoplanets through transit or eclipse observations, the large contrast between the stellar and planetary emission (e.g., about a thousand times in the infrared for a hot Jupiter around an FGK dwarf star) make the data analysis an intrinsically challenging task. For example, for the Spitzer Space Telescope, most planetary signals (e.g., Stevenson et al. 2010, Demory et al. 2012) lie below the instrument's design criteria for photometric stability (Fazio et al. 2004). Extracting planetary signals at this precision requires meticulous data reduction. Despite our best attempts to account for all known systematics, time-correlated residuals (or red noise) between the data and models often remain. These systematics may originate from instrumental or astrophysical sources, for example: stellar flux variations from flares or granulation; imperfect flat fielding; or telluric variations from changing weather conditions, differential extinction, or imperfect telescope systematics corrections from changing

patricio.cubillos@oeaw.ac.at telescope pointing. Many authors have acknowledged correlated noise as an important source of noise in time-series datasets (e.g., Pont et al. 2006, Winn et al. 2007, Agol et al. 2010, Cubillos et al. 2013).

Correlated noise affects both the accuracy and the precision of estimates of the model parameters. The typical statistical analyses neglect the correlation between data points (e.g., likelihood functions such as $\chi^{2}$, based on uncorrelated noise). Hence, their estimated best-fitting values may be biased, whereas their credible regions (Appendix A) can be incorrect. This paper reports our study of three common correlated-noise estimators found in the exoplanet literature; specifically, in analyses of transit and eclipse exoplanet light curves. First, the time-averaging method (Pont et al. 2006, Winn et al. 2007) compares the standard deviation of the data to the (expected) uncorrelated-noise standard deviation, scaling the uncertainties accordingly. Next, the residualpermutation (or "prayer bead") method (Bouchy et al. 2005) uses a data-shifting (bootstrap-like) algorithm that preserves the structure of the residuals. Lastly, the method of Carter \& Winn (2009) calculates the likelihood function in a wavelet basis, where the correlation between the wavelet coefficients is negligible. Qualitatively speaking, these methods do return 
larger parameter uncertainties for stronger correlated noise. However, besides Carter \& Winn (2009), there are few efforts to validate their quantitative accuracy. We have implemented these methods, testing them with real and synthetic exoplanet eclipse data.

Although recently there has been development of additional sophisticated methods to model exoplanet light curves (e.g., Gibson et al. 2012, Jordán et al. 2013, Morello 2015, Evans et al. 2015), we explicitly exclude them from this study because the large number of runs and dataset sizes would require unfeasible amounts of computing power. For example, Gaussian processes can become computationally prohibitive for datasets larger than $\sim 1000$ data points (Gibson 2014).

With a focus on atmospheric characterization, we concentrate on estimating the eclipse depths from Spitzer exoplanet light curves, since they represent the largest and best-quality sample of exoplanet data beyond $2 \mu \mathrm{m}$. Spitzer data are affected by two well-known systematics: time-varying sensitivity (ramp) and intra-pixel sensitivity variations (Knutson et al. 2009, Charbonneau et al. 2005). Although several models have been proposed to correct for these systematics (Harrington et al. 2007, Knutson et al. 2008, Ballard et al. 2010, Agol et al. 2010, Stevenson et al. 2012a, Lewis et al. 2013, Deming et al. 2014), the corrections are not always perfect, and thus many light-curve fits exhibit time-correlated residuals.

In Section 2, we discuss the impact of correlated noise on determining model-parameter uncertainties. In Section 3 , we review the most commonly used correlated-noise estimators in exoplanet analyses. In Section 4, we test and compare the correlated-noise methods by retrieving synthetic eclipse curves that were injected into synthetic and real lightcurve data. In Section 5 we present our open-source package, Multi-Core Markov-Chain Monte Carlo $\left(\mathrm{MC}^{3}\right)$, to calculate the model-parameters' credible regions. Finally, in Section 6 we present our conclusions.

\section{THE IMPACT OF CORRELATED NOISE}

A central ingredient in both frequentist and Bayesian parametric modeling is the sampling distribution for the data: the joint probability density function (PDF) for the data values, as a function of the model parameters. In our time series setting, we denote the data by $\vec{y}=\left(y_{1}, \ldots, y_{n}\right)$, with $y_{i}$ denoting the value of a measurement at time $t_{i}$. Similarly, we denote the model predictions by $\vec{f}=\left(f_{1}, \ldots, f_{n}\right)$, with $f_{i}=f\left(t_{i} ; \theta\right)$ for a model function with parameters $\theta$. Note that the predictions are functions of the parameters, $f_{i}(\theta)$, but we often suppress the parameter dependence for convenience. The sampling distribution is the $n$-dimensional joint PDF, $p(\vec{y} \mid \theta)$.

When $\vec{y}$ is fixed to an actually observed data vector, the sampling distribution as a function of the model parameters is called the likelihood function, $\mathcal{L}(\theta)$. Bayesian methods quantify uncertainty in the parameters via the dependence of $\mathcal{L}(\theta)$ on the parameters; Bayes's theorem and the law of total probability convert this dependence into posterior probabilities for statements about the parameters. Frequentist methods quantify uncertainty by first defining statistics (functions of $\vec{y})$ that produce point estimates or intervals in the parameter space (perhaps using the $\theta$ dependence of the likelihood function), and then using the sampling distribution to quantify the variability of the statistics across ensembles of hypothetical data vectors. The variability in the sample space then is mapped into uncertainty quantifications in the parameter space (e.g., bias of a point estimate, or coverage of a confidence interval).

Commonly, the data are modeled as the sum of the predictions and independent, zero-mean, normally-distributed noise,

$$
y_{i}=f_{i}(\theta)+\epsilon_{i},
$$

with independent noise probabilities

$$
p\left(\epsilon_{i}\right)=\frac{1}{\sigma_{i} \sqrt{2 \pi}} \exp \left[-\frac{\epsilon_{i}^{2}}{2 \sigma_{i}^{2}}\right],
$$

where $\sigma_{i}$ is the standard deviation for the noise contribution in measurement $i$. In this scenario, the sampling distribution factors,

$$
\begin{aligned}
p(\vec{y} \mid \theta) & =\prod_{i} p\left(y_{i} \mid \theta\right) \\
& =\frac{1}{(2 \pi)^{n / 2}}\left(\prod_{i} \sigma_{i}\right)^{-1} \exp \left[-\frac{1}{2} \sum_{i} \frac{\epsilon_{i}(\theta)^{2}}{\sigma_{i}^{2}}\right]
\end{aligned}
$$

where the sum inside the exponential is the familiar " $\chi^{2}$ " sum of squared, standardized residuals. Parameter estimation based on maximizing Eq. (3) with respect to $\theta$ is called weighted least squares (WLS) regression when $f_{i}(\theta)$ is linear with respect to the parameters. If in addition the $\sigma_{i}$ values are all the same (homoskedastic), the approach is called ordinary least squares (OLS).

In the general case, correlated Gaussian noise has a multivariate normal PDF with a non-diagonal precision matrix $K$,

$$
p(\epsilon)=\frac{|K|^{1 / 2}}{(2 \pi)^{n / 2}} \exp \left[-\frac{1}{2} \sum_{i j} \epsilon_{i} K_{i j} \epsilon_{j}\right],
$$

where $|K|$ denotes the determinant of the precision matrix. The derivation to the specific case of uncorrelated Gaussian noise is trivial. As a consequence of independence, $K$ becomes a diagonal matrix with components

$$
K_{i j}=\frac{1}{\sigma_{i} \sigma_{j}} \delta_{i j} .
$$

Therefore, the sum in Eq. (4) collapses to the single sum in Eq. (3).

Parameter estimation based on maximizing this likelihood function with respect to $\theta$ is called generalized least squares (GLS) regression when $f_{i}(\theta)$ is linear with respect to the parameters. When the model has nonlinear dependence on any of the parameters, maximum likelihood estimate is the nonlinear least squares estimate, regardless of whether the errors are correlated or homoskedastic.

Methods currently used to handle correlated noise fall into two broad classes. Methods like the Carter \& Winn (2009) wavelet approach estimate a correlation matrix, and produce 
GLS estimates. In contrast, the time-averaging and residualpermutation approaches rely on WLS for estimation, but devise rules for inflating uncertainties to account for the ignored correlations. It is well known that non-linear least squares estimates are statistically consistent; that is, asymptotically (as $n \rightarrow \infty)$, estimates converge to the true parameter values (Wu 1981). Roughly speaking, although correlation complicates the way information accumulates across samples, infinite sample size ameliorates the complications. However, since WLS does not account for correlations, the quality of estimates can be significantly compromised with finite sample size.

In Appendix B we describe two simple example calculations comparing WLS and GLS that provide some insight into the costs of ignoring noise correlation. The first example treats estimation of the amplitude of a constant signal in the presence of autoregressive noise, a simple and analytically tractable example of correlated noise. When noise is independent, with standard deviation $s$, the uncertainty in an estimate of a constant signal level is $s / \sqrt{n}$, the familiar "root- $n$ " law. The WLS estimate has just this behavior. In contrast, when the noise is known to be positively correlated between adjacent samples, the uncertainty in the GLS estimate decreases more slowly than $1 / \sqrt{n}$. This kind of example motivates approaches like time averaging that attempt to account for correlation merely by inflating uncertainties.

The second example replaces the constant signal with an eclipse-like dip signal. The dip location and width are presumed known; the background level and dip depth are to be estimated. In this case, a simple simulation study shows that noise correlation does not merely inflate uncertainties. It can also corrupt parameter estimates, with WLS estimates potentially taking values far away from the optimal estimates that account for noise correlations. This occurs when parameters of interest pertain to temporally localized structure in the model, for which noise correlations can significantly change the data projections needed for accurate inference.

Together, these examples show that methods that seek to account for correlations only by inflating parameter uncertainties are at best suboptimal (producing larger estimation errors than could be achieved with a good correlated noise model), and can sometimes be significantly misleading.

\section{COMPUTING PARAMETER UNCERTAINTIES}

\subsection{Markov-chain Monte Carlo}

In the Bayesian framework, a credible region for the parameters of a model, $\mathcal{M}$, can be computed via the Markovchain Monte Carlo (MCMC) algorithm. The MCMC method generates a large number of random samples from the parameter space with a probability density proportional to the posterior probability distribution:

$$
p(\theta \mid \vec{y}, \mathcal{M}) \propto p(\theta \mid \mathcal{M}) p(\vec{y} \mid \theta, \mathcal{M}),
$$

where $p(\theta \mid \mathcal{M})$ is the prior probability distribution. A marginal highest-posterior-density (HPD) credible region for each parameter is then obtained from the interval that contains a certain fraction of the highest posterior density (typically $68 \%, 95 \%$, or $99 \%$ ) of the marginalized posterior (see
Appendix A). For example, when the posterior follows a normal distribution, the $68.3 \%$ marginal credible interval corresponds to the interval contained within one standard deviation from the mean.

Inference based on the likelihood function of Eq. (3) works well when the noise contributions are independent and normally distributed; however, it does not account for timecorrelated noise. Alternatively, an inference that uses the full covariance matrix, as in of Eq. (4), should account for correlated noise, although its calculation often becomes computationally prohibitive.

\subsection{Time Averaging}

Pont et al. (2006) developed a method to compute the uncertainty of a transit or eclipse-depth estimation using the light-curve data points themselves. They considered the noise as the sum in quadrature of two components, a purely white (uncorrelated) source (characterized by a standard deviation per data point $\sigma_{w}$ ), and a purely time-correlated source (characterized by $\sigma_{r}$ ). Pont et al. (2006) assumed the white-noise component to scale as $\sigma_{w} / \sqrt{n}$, with $n$ the number of data points in the transit; whereas the time-correlated standard deviation, $\sigma_{r}$, to be independent of the number of data points. Then for any given signal, the uncertainty of a measurement should scale as:

$$
\sigma_{d}=\sqrt{\frac{\sigma_{w}^{2}}{n}+\sigma_{r}^{2}} .
$$

For small $n, \sigma_{d}$ may be dominated by $\sigma_{w} / \sqrt{n}$, whereas as $n$ increases, $\sigma_{d}$ approaches $\sigma_{r}$. The time-averaging method uses this fact to estimate the contribution from the correlated noise. Note, however, that this hypothesized behavior is not typical of stationary correlated noise models exhibiting longrange dependence, which instead have $\sigma_{d}$ decreasing at a rate slower than $1 / \sqrt{n}$, but still monotonically decreasing to zero (Beran et al. 2013).

We implement the time-averaging procedure as described by Winn et al. (2007). First, we calculate the residuals between the data points and the best-fitting model. Then, we group the residuals in time-ordered, non-overlapping bins of $N$ elements each, and calculate their mean values. Lastly, we calculate the standard deviation (or root mean squared, rms) of the binned residuals, $\mathrm{rms}_{N}$. We repeat the process for a range of bin sizes from one to half the data size. The uncertainty of $\mathrm{rms}_{N}$ is approximately $\sigma_{\mathrm{rms}}=\mathrm{rms}_{N} / \sqrt{2 M}$ (see Appendix C).

Now, let $\sigma_{1}$ be the rms value of the non-binned residuals (presumed to be dominated by white noise). In the absence of correlated noise, the expected rms for the set of $M$ bins, each containing $N$ points, is given by the extrapolation of $\sigma_{1}$ (Winn et al. 2008):

$$
\sigma_{N}=\frac{\sigma_{1}}{\sqrt{N}} \sqrt{\frac{M}{M-1}} .
$$

The $\mathrm{rms}_{N}$ and $\sigma_{N}$ curves are analogous to $\sigma_{d}$ and $\sigma_{w}$, respectively. The time-averaging correction inflates the data uncertainties multiplying them by the ratio $\beta_{N}=\mathrm{rms}_{N} / \sigma_{N}$ if $\beta_{N}$ is statistically larger than one (i.e., by more than 


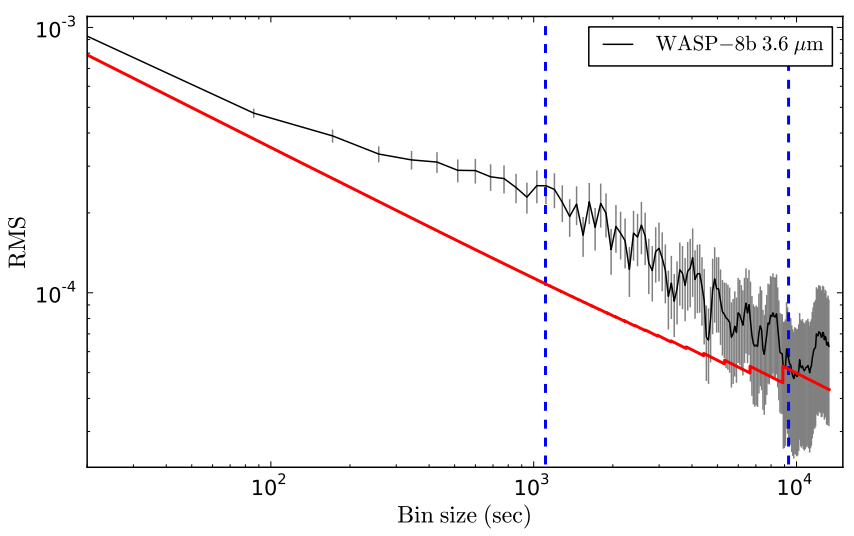

Figure 1. Binned residuals rms vs. bin size (black curve with gray error bars) of WASP-8b Spitzer eclipse at $3.6 \mu \mathrm{m}$ (PI J. Harrington, Program ID 60003, see Cubillos et al. 2013). The red curve corresponds to the expected rms for white noise (Equation 8). The sawtooth look of the curve arises from the discreet change in $M$, which becomes more significant as $N$ increases. The vertical dashed lines mark the duration of ingress/egress (left) and eclipse (right). The gray vertical error bars denote the $1 \sigma$ uncertainty of the rms residuals $\left(\operatorname{rms}_{N} / \sqrt{2 M}\right)$.

$\left.1 \sigma_{\beta}=\sigma_{\mathrm{rms}} / \sigma_{N}\right)$. Finally, one runs a white-noise MCMC analysis, i.e., with Eq. (3), using the inflated data errors. One typically visualizes both curves in an rms vs. bin size plot (Figure 1).

\subsubsection{Behavior at Large Bin Sizes}

One has to be aware that the binned-rms uncertainty $\sigma_{\text {rms }}$ is an asymptotic approximation. In the large bin-size regime (equivalently, small $M$ ) that approximation is not justified. The reason is that the marginalized posterior distribution for $\operatorname{rms}_{N}$ (Eq. 33), which has the form of an inverse-gamma distribution, becomes increasingly skewed as $M$ decreases.

By comparing the $68 \%$-credible-region error bars of the inverse-gamma formula with the asymptotic approximation, we find that the latter moderately overestimates the lower error bar by $5 \%-10 \%$ for $M<200$. For the upper error bar the asymptotic approximation underestimates the error by $5 \%$ to $450 \%$ between $M=200$ and $M=2$. If one does not consider the skewed error bars, it may seem that the rms curves deviate below the $\sigma_{N}$ curve at large bin sizes (e.g., Stevenson et al. 2012a, Cubillos et al. 2013, Blecic et al. 2013). However, this deviation is not statistically significant when one computes the uncertainties with the correct posterior distribution.

Since the typical transit (or eclipse) observation does not last much longer than the duration of the transit itself (usually one-two hours of out-of-transit before and after), it is important to consider the asymmetric rms error bars to properly account for correlated noise. Furthermore, since the signalto-noise ratio of $\mathrm{rms}_{N}$ decreases proportionally to $\sqrt{M}$ as $N$ increases, one ideally wants the longest-possible observation duration to improve the constraint on $\beta_{N}$ at the desired timescale of the event.

\subsection{Residual Permutation}

Residual permutation (also called the prayer bead method) is inspired by nonparametric bootstrapping methods from frequentist statistics. Nonparametric bootstrap methods directly use the sampled data (typically via resampling) to generate a distribution that approximates the sampling distribution, $p\left(\vec{y} \mid \theta^{*}\right)$, for the true parameter values, $\theta^{*}$. Nonparametric bootstrapping typically relies on independent resampling of the data or residuals (possibly re-scaled), with replacement (e.g., Davison \& Hinkley 1997, Ruppert \& Matteson 2015).

The motivating idea of the residual-permutation approach is to shift the data while preserving the time ordering and, thus, preserving the correlation structure. While the structure is indeed preserved, the residual-permutation method does not resample with replacement, a crucial requrement for nonparametric bootstrapping to work, i.e., to produce independent replicated datasets (a resample of the entire time-series observation). When there is correlated noise, the shifted datasets do not correspond to an independent resampling from any distribution, and thus do not exhibit the variability necessary for correct uncertainty quantification (e.g., computing confidence levels or estimator bias).

In the exoplanet field, the residual-permutation technique has been repeatedly used to estimate parameter uncertainties. However, the name of the technique has been loosely used to describe similar, but not equivalent procedures over the past decade. Bouchy et al. (2005), Gillon et al. (2007), and Southworth (2008) all describe different methods, when referring to residual permutations. Some authors reference Jenkins et al. (2002), who actually use a "segmented bootstrap", applying the method for detection instead of parameter estimation. Furthermore, several authors have wrongly attributed the method to Moutou et al. (2004). Thus, there is a visible lack of rigor in the use of this method.

Currently, the most widely-used version of residual permutation is the one described by Southworth (2008) or Winn et al. (2008). This implementation computes the residuals between the light curve and the best-fitting model, cyclically shifts the residuals (preserving the point-to-point structure and thus the "redness" of the noise) by a given number of data points, adds the residuals back to the model, and finds a new set of best-fitting parameters. Usually, either one repeats the shift-fit process for a large number of iterations with random shifts, or one sequentially shifts the residuals by one data point at a time, fitting all possible shifts. Each parameter uncertainty is then given by the respective standard deviation of the distribution of best-fitting values. As already noted, this does not correspond to a sound resampling procedure, thus we will not consider residual permutations for the subsequent analyses.

There is a significant literature on generalizing the independent and identically distributed nonparametric bootstrap idea to address time series problems with correlated noise; this is a topic of ongoing research. One widely used approach is the block bootstrap. Presuming the investigator knows or can estimate a longest scale for correlations, $\Delta t$, the data are divided into blocks of length greater than $\Delta t$, and bootstrap resampling is done by drawing blocks of data at random to build a replication. A particular block rigidly preserves the time ordering of a subset of the data; in replications, it will appear shifted in time by various amounts. This 
behavior resembles the behavior of the prayer bead method. But block resampling produces greater variability than shifting the entire data vector, and by sampling with replacement, it produces ensembles that approximate independent draws from a (dependent) sampling distribution. The "segmented bootstrap" devised by Jenkins et al. (2002) for analysis of ground-based transit photometry is similar to the block bootstrap. The block bootstrap only works if the correlation scale is significantly shorter than the span of the data, which will often not be true for Spitzer exoplanet eclipse data, so we do not consider it further here. Further details about the block bootstrap and other methods for resampling dependent data may be found in Lahiri (2003).

\subsection{Wavelet Analysis}

Carter \& Winn (2009) introduced to the exoplanet field a technique where the time-correlated noise is modeled using wavelet transforms (Deriche \& Tewfik 1993, Wornell 1993, Wornell \& Oppenheim 1992a,b). This method projects the time series residuals into an orthonormal wavelet basis, where the off-diagonal terms of the covariance matrix become negligible, thus simplifying the likelihood function calculation. Furthermore, they assumed noise that has a power spectral density with frequency $f$, varying as $1 / f^{\gamma}$. They parameterized the noise with three parameters, $\gamma, \sigma_{\omega}$, and $\sigma_{r}$, as described in Equations (41)-(43) of Carter \& Winn (2009).

A thorough review of wavelets is beyond the scope of this work; see Mallat (2008) and Wornell (1996) for more comprehensive discussions. Briefly, a wavelet transform projects a time-series signal onto a basis of functions that are dilations and translations of a compact parent ("wavelet") function. The resulting transform has two dimensions, scale and location (in time). The discrete wavelet transform (DWT) consists of the hierarchical application over $M$ dilation scales of an orthonormal wavelet transform on a discrete time-series signal. For a signal consisting of $N=N_{0} 2^{M}$ uniformlyspaced samples (with $N_{0}$ integer), and a wavelet function with $2 N_{0}$ coefficients, the DWT produces $N_{0}$ scaling coefficients and $N_{0} 2^{m-1}$ wavelet coefficients at each scale $m$, totaling $N_{0}\left(2^{M}-1\right)$ wavelet coefficients.

Carter \& Winn (2009) recommend the fourth-order Daubechies wavelet basis (Daubechies 1988) for modeling time-series correlated noise, which we adopt in the current work. This is a basis well localized in time and frequency, well suited for $1 / f^{\gamma}$ noise (Wornell 1996). Carter \& Winn (2009) found that correlations between the wavelet and scaling coefficients decays faster for the Daubechies basis than the Haar basis, producing negligible covariances. Another advantage is that since the Daubechies basis is well localized in time, it reduces artifacts arising from the assumption of a periodic boundary condition by the wavelet transform.

\subsubsection{Wavelet-based Likelihood}

The likelihood function in the wavelet analysis is calculated in the following way. Let $\epsilon(t)$ be the fitting residuals of a time-series signal. Considering $\epsilon(t)$ as the contribution of a time-correlated $(\gamma \neq 0)$ and an uncorrelated $(\gamma=0)$ component:

$$
\epsilon(t)=\epsilon_{\gamma}(t)+\epsilon_{0}(t),
$$

this method calculates the DWT of $\epsilon(t)$ to produce the wavelet, $r_{n}^{m}$, and scaling, $\bar{r}_{n}^{1}$, coefficients of the signal. The variances of these coefficients are computed, respectively, as:

$$
\begin{aligned}
\sigma_{W}^{2} & =\sigma_{r}^{2} 2^{-\gamma m}+\sigma_{\omega}^{2} \\
\sigma_{S}^{2} & =\sigma_{r}^{2} 2^{-\gamma} g(\gamma)+\sigma_{\omega}^{2},
\end{aligned}
$$

where $\sigma_{\omega}$ and $\sigma_{r}$ parameterize the standard deviation of the uncorrelated and the correlated-noise signals, respectively, and $g(\gamma)=1 /\left(2^{1-\gamma}-1\right)$ for $\gamma \neq 1$ (following derivations from, e.g., Fadili \& Bullmore 2002, Wornell 1993) and $g(\gamma)=1 / 2 \ln 2$ for $\gamma=1$ (Carter \& Winn 2009) (see Appendix ??). Therefore, the wavelet-based likelihood function is given by

$$
\begin{aligned}
\mathcal{L}\left(\mathbf{x}, \sigma_{\omega}, \sigma_{r}\right)= & \left\{\prod_{m=1}^{M} \prod_{n=1}^{N_{0} 2^{m-1}} \frac{1}{\sqrt{2 \pi \sigma_{W}^{2}}} \exp \left[-\frac{\left(r_{n}^{m}\right)^{2}}{2 \sigma_{W}^{2}}\right]\right\} \times \\
& \left\{\prod_{n=1}^{n_{0}} \frac{1}{\sqrt{2 \pi \sigma_{S}^{2}}} \exp \left[-\frac{\left(\bar{r}_{n}^{1}\right)^{2}}{2 \sigma_{S}^{2}}\right]\right\} .
\end{aligned}
$$

Equation (12) allows one to fit a model, sample its parameter's posterior distribution, and determine the credible regions, while taking into account the effects of time-correlated noise.

During our review and implementation of the waveletlikelihood technique from Carter \& Winn (2009), we found a few oversights in their equations and code (available in the Astronomical Source Code Library, ASCL ${ }^{1}$ ). See details in Appendix D.

\section{CORRELATED-NOISE TESTS FOR EXOPLANET ECLIPSE DATA}

To assess the performance of the correlated-noise estimators described in Section 3, we carried out injection-retrieval eclipse simulations. We focus on estimating the secondaryeclipse depth in a light curve observation, creating synthetic light curves that resemble Spitzer InfraRed Array Camera (IRAC) observations in terms of the signal-to-noise ratio (S/N), known systematics, cadence, observation duration, and eclipse shape.

In our first experiment we test the estimators' performances when the time-correlated noise is described by a stochastic wavelet signal with a $1 / f$ power spectral density (similar to the experiment of Carter \& Winn 2009). We test the case when the observation time span is similar to the eclipse-event duration (Section 4.1, typical of real Spitzer secondary eclipse observations) and for the hypothetical case when the time span lasted an order of magnitude longer than the eclipse event (Section 4.2). In a second experiment (Section 4.3) we test the estimators on a more realistic case by injecting a synthetic eclipse signal into Spitzer phase-curve datasets.

\subsection{Synthetic-noise Simulation}

\footnotetext{
${ }^{1}$ http://asterisk.apod.com/viewtopic.php?f=35\&t=21675
} 

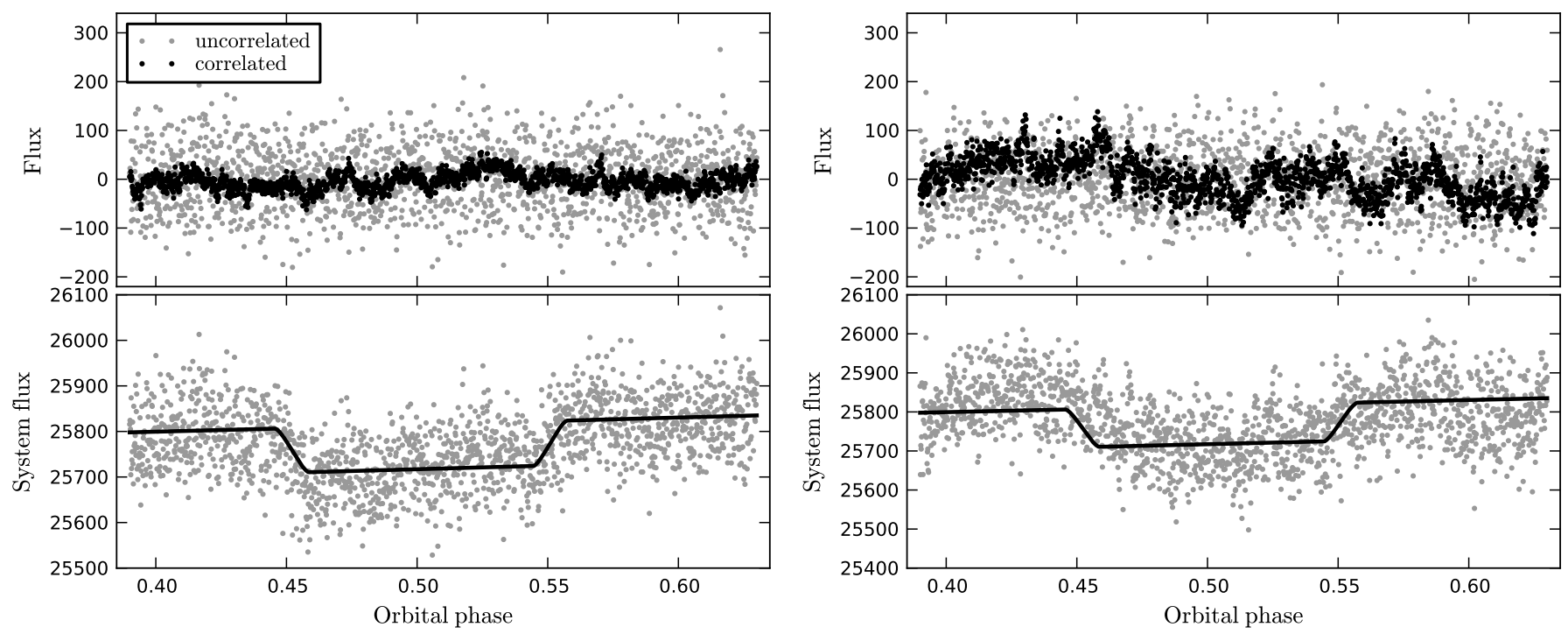

Figure 2. Simulated Spitzer time-series datasets. The top panels overplot the correlated (black) and uncorrelated (grey) noise components of the light curves vs. orbital phase. The bottom panels show the synthetic light curves (eclipse, ramp, and noise) vs. orbital phase in gray. The black solid line shows the noiseless model. The noise rms ratios are $\alpha=0.25$ (left panels) and $\alpha=0.5$ (right panels).

In this simulation we generate synthetic light curves by combining a Mandel \& Agol (2002) eclipse model, a linear ramp model, and a signal with both correlated and uncorrelated noise. The light-curve parameters closely follow those of a Spitzer observation of the WASP-12 system (Table 1). The signal consists of 1700 data points, with a cadence of $\sim 12$ seconds between data points, spanning an orbital-phase range from 0.39 to 0.63 , about twice the eclipse duration.

Table 1. Synthetic light curve parameters

\begin{tabular}{lc}
\hline \hline Parameter & Value \\
\hline Eclipse depth (counts) & 98.1 \\
Eclipse duration (phase) & 0.1119 \\
Eclipse mid point (phase) & 0.5015 \\
Eclipse ingress/egress time (phase) & 0.013 \\
Ramp slope (counts/phase) & 0.006 \\
System flux (counts) & 25815 \\
$\sigma_{\omega}$ (counts) & 64.5 \\
$\sigma_{r}$ (counts) & 0,230, and 459 \\
\hline
\end{tabular}

We created three sets of 5000 light-curve realizations each. For each realization, we generate a zero-mean random normal distribution, which we add to the light curve as the uncorrelated noise signal. We adjust the variance of this signal $\left(\sigma_{\omega}^{2}\right)$ to yield an eclipse-depth signal-to-noise ratio of 30. Additionally, we generate purely-correlated $1 / f$ signals $\left(\sigma_{\omega}=0\right)$ using a Gaussian random number generator to produce wavelet coefficients with variances given by Equations (10) and (11). Then, we apply the inverse DWT to transform the signal from the wavelet basis to the time domain. Following the notation of Carter \& Winn (2009), we denote by $\alpha$ the ratio between the rms of the uncorrelated and correlated noise signals.

We constructed the signals in each of the three sets to have a pure uncorrelated noise, a weak time-correlated sig- nal, and a strong correlated signal $(\alpha=0.0,0.25$, and 0.5 , respectively). Figure 2 shows two synthetic light curves for $\alpha=0.25$ and 0.5 . Note that our designations of "weak" and "strong" are, to some extent, arbitrary. We selected these limits based on our experience and tests: for $\alpha \lesssim 0.20$, the time-correlated signal becomes negligible compared to the uncorrelated-noise signal, whereas values of $\alpha \sim 0.5$ are on the level of what we have observed in some cases (e.g., WASP-8b, Cubillos et al. 2013).

For each realization, we compute the parameter posteriors using the methods described in Section 3, excluding residual permutation, which we deem to be unsound. Our modelfitting routines only fix the eclipse ingress/egress-time parameter (usually poorly-constrained by eclipse data), leaving free the system flux, eclipse depth, eclipse midpoint, eclipse duration, and ramp slope. First, we carry out a "white analysis" (i.e., ignoring the time-correlation between data points) by using Equation (3) to compute the model-parameter bestfitting values (using the Levenberg-Marquardt algorithm) and their posterior distributions (using a MCMC).

Next, we use the best-fit results to calculate the timeaveraging rms-vs.-bin size curves. We retrieve the $\beta$ factor at three timescales: at the ingress time, at the eclipse duration, and at the time of maximum $\beta$ ( $\beta_{\max }$, Figure 3$)$. In accordance with the discussion in Section 3.2.1, most $\beta$ values at the eclipse-duration timescale (similar to the total observation duration) were not significant. Thus, we adopted $\beta_{\max }$ as the scaling factor to calculate the time-averaging method uncertainties. Finally, we apply the wavelet-based likelihood method in an MCMC guided by Equation (12), simultaneously fitting the noise parameters $\left(\sigma_{\omega}\right.$ and $\left.\sigma_{r}\right)$ and the model parameters, while keeping $\gamma$ fixed at 1 . We find that a noninformative logarithmic prior on $\sigma_{r}$ handle the case with no correlated noise better than a flat prior. A log-flat prior is a scale-invariant prior that has an equal probability per order of magnitude. This is a more convenient prior when the parameter may range over several orders of magnitude (Gre- 

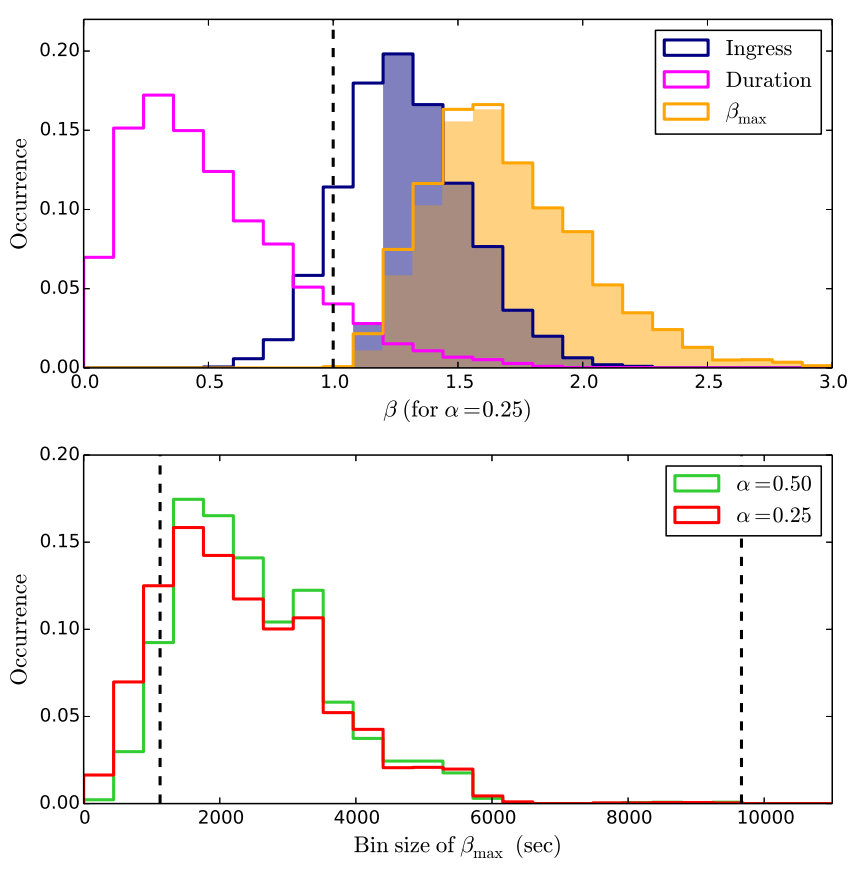

Figure 3. Top: Normalized distribution of $\beta$ for the $\alpha=0.25$ set. The histograms represent $\beta$ measured at the ingress-time and eclipse-duration timescales, and at the maximum value of $\beta$. The vertical dashed line marks $\beta=1$. The colored shaded areas denote the corresponding fraction of trials that were more than $1 \sigma_{\beta}$ greater than one. The distributions for the other two sets $(\alpha=0$ and 0.5$)$ were similar. Bottom: Normalized distribution of the bin sizes for $\beta_{\max }$. The vertical dashed lines indicate the ingress time and eclipse duration.

gory 2005). The only requirement is that the parameter value must be positive.

\subsubsection{Results}

To assess the quality of the inferences from the time averaging and wavelet likelihood methods, we performed calibration tests, i.e., tests of the repeated-sampling (frequentist) performance of the inferences, when applied across an ensemble of simulated datasets.

The first test computes a measure of the relative accuracy of the eclipse depth estimates (i.e., accuracy relative to the reported uncertainty), also known as "number-of-sigma" statistic as described by Carter \& Winn (2009). The simulated datasets are large enough that the marginal posterior PDFs for the eclipse depth are typically nearly normal. This motivates measuring relative accuracy by the number of posterior standard deviations between the best-fit value and the true value used for a simulation,

$$
\mathcal{N}_{p}=\frac{\hat{p}-p}{\sigma_{p}}
$$

where $p$ is the true eclipse depth used for the simulation, $\hat{p}$ is the best-fit estimate, and $\sigma_{p}$ is the standard deviation of the marginal posterior for $p$. If the marginal posterior were normal, and if $\sigma_{p}$ were constant across the simulations, we would expect $\mathcal{N}_{p}$ to have normal standard deviation with mean $\left\langle\mathcal{N}_{p}\right\rangle$ zero, and standard deviation $\sigma_{\mathcal{N}}$ unity. In principle, a departure of $\left\langle\mathcal{N}_{p}\right\rangle$ from zero would suggest a lack of accuracy of an analysis, whereas a departure of $\sigma_{\mathcal{N}}$ from unity would suggest an under- or overestimated precision $\left(\sigma_{\mathcal{N}}>1\right.$ or $\sigma_{\mathcal{N}}<1$, respectively). In general, however, neither condition rigorously holds; the posteriors are slightly non-normal, and the value of $\sigma_{p}$ varies a bit. As a result, we do not expect $\mathcal{N}_{p}$ to have a standard normal distribution exactly (even for many simulations). Nevertheless, the mean and shape of the $\mathcal{N}_{p}$ distribution can reveal significant calibration failures of inferences.

The second test examines the conditional coverage of marginal credible regions for the eclipse depth. For a set of simulations with parameters $\theta$, we compute $C_{Q}(\theta)$, the coverage of marginal credible regions for the eclipse depth that were computed to contain a fraction $Q$ of the posterior probability. That is, we fix a size for the eclipse depth credible region to be tested (say, $Q=0.683$ for a conventional " $1 \sigma$ " region), and we compute the fraction of times the credible region contains the true eclipse depth value.

Some caution is required in interpreting results of conditional coverage tests. Bayesian credible regions will not in general be perfectly calibrated for fixed $\theta$. Rather, Bayesian methods produce regions with exact average coverage, i.e., with $\left\langle C_{Q}(\theta)\right\rangle=Q$ if one averages over the prior. The bottom line of these considerations is that, for a set of simulations with a fixed set of true parameter values, we do not expect $C_{Q}(\theta)$ to equal $Q$ exactly. But large departures from $Q$ likely indicate problems with an inference procedure. It is possible, at least in principle, to more thoroughly verify calibration of Bayesian MCMC algorithms (e.g., to average over $\theta$, or to consider all possible sizes of credible regions; see Cook et al. 2006), but we focus on simpler conditional tests here. For the coverage calculations reported below, we used the $68.3 \%$ marginal highest posterior density credible region.

Figure 4 shows the $\mathcal{N}_{\mathrm{p}}$ distributions for each method and dataset. Table 2 shows the mean and standard deviation of $\mathcal{N}_{\mathrm{p}}$ and coverage fraction $C_{Q=0.68}$ (i.e., the fraction of trials where the $68 \%$ HPD covers the true depth value). The white analysis of the uncorrelated-noise set serves as a control sample. As expected, the $\mathcal{N}_{p}$ distribution for this case shows a negligible deviation from zero, a standard deviation close to one, and a $68 \%$-HPD coverage fraction of $\sim 68 \%$. The correlated-noise runs reveal the failure of the white analysis to account for correlated noise; as the correlated-noise component increases, the mean and standard deviation of $\mathcal{N}_{p}$ increase (suggesting a decrease of accuracy and underestimated uncertainties), which is well correlated with the lower coverage fraction.

The time-averaging method seems to improve the precision for the correlated-noise runs with respect to those of the white analysis (less underestimated uncertainties), as shown by the smaller $\sigma_{\mathcal{N}}$. Accordingly, the coverage fractions closer to $68 \%$ indicate an improvement in the parameter estimation. Note that the time-averaging method does not affect the accuracy (with respect to the white analysis). Since all the data uncertainties are inflated by a common scaling factor, the best-fitting solution does not change. Therefore, the variation in $\left\langle\mathcal{N}_{p}\right\rangle$ is a consequence of the variation in the precision and any possible underlying correlation between precision and accuracy for the sample. 

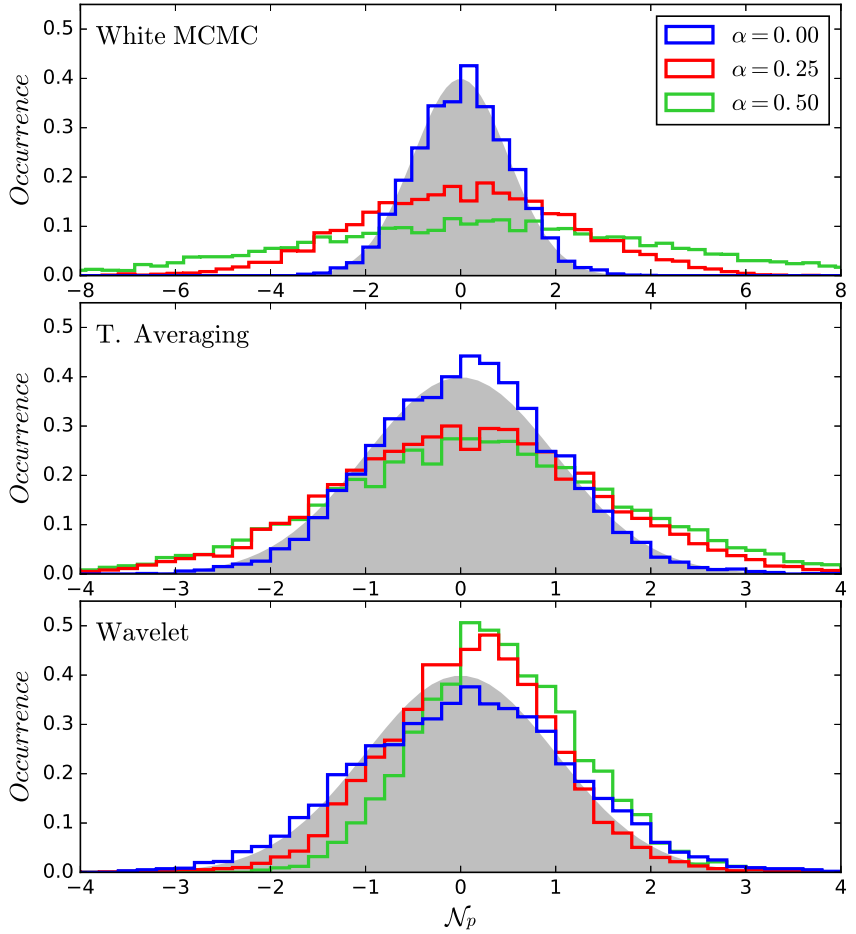

Figure 4. Eclipse-depth histogram of the relative-accuracy statistic for synthetic-data simulations. The top, middle, and bottom panels show the results for the white-MCMC, wavelet-likelihood, and time-averaging methods, respectively. The color code denotes the sample (see legend). The background gray contour denotes a standard normal distribution for comparison. All histograms are normalized such that the integral of each curve adds to one.

The wavelet method also seems to improve the parameter estimation of the correlated samples with respect to the white analysis. However, this time the method seems to overestimate the uncertainties, as shown by the coverage fractions greater than $68 \%$ and the values of $\sigma_{\mathcal{N}}$ smaller than one. The values of $\left\langle\mathcal{N}_{p}\right\rangle$ lie at the same level as those of the white analysis.

Overall, both the time-averaging and wavelet methods improve the eclipse-depth estimation over a white analysis. For a sample size of 3000 trials, the coverage uncertainty is roughly $2 \%$ (from a root- $N$ estimate). Since we carried out only a conditional study, there is an additional error budget to consider. Only a substantial mismatch between coverage and credible region size would be evidence that there is a problem. The results of the wavelet analysis may be evidence of a real coverage mismatch, but it is not at a level that would be surprising for conditional vs. average coverage. If real, this emphasizes the challenges of retrieving reliable parameter estimates from light curves affected by correlated noise, considering that we generated the synthetic signal with wavelet function.

\subsection{Synthetic-noise for Long-duration Simulation}

Here we describe tests of the time-averaging method for datasets long enough such that the eclipse duration lies at timescales where the asymptotic approximation is still valid. To do so, we replicate the previous simulation (synthetic transit, white noise, and $1 / f$ noise signals) for an observation last-
Table 2. Relative-accuracy Statistics and Coverage Fraction for Synthetic-data Simulations

\begin{tabular}{llll}
\hline \hline Estimation method & $\left\langle\mathcal{N}_{p}\right\rangle$ & $\sigma_{\mathcal{N}}$ & $C_{0.68}$ \\
\hline White MCMC & & & \\
$\quad \alpha=0.00$ & 0.048 & 1.008 & 0.67 \\
$\alpha=0.25$ & 0.091 & 2.230 & 0.33 \\
$\quad \alpha=0.50$ & 0.420 & 3.826 & 0.21 \\
Time Averaging & & & \\
$\quad \alpha=0.00$ & 0.044 & 0.954 & 0.71 \\
$\quad \alpha=0.25$ & 0.062 & 1.418 & 0.52 \\
$\quad \alpha=0.50$ & 0.177 & 1.581 & 0.48 \\
Wavelet & & & \\
$\quad \alpha=0.00$ & 0.056 & 1.128 & 0.63 \\
$\alpha=0.25$ & 0.111 & 0.897 & 0.74 \\
$\alpha=0.50$ & 0.408 & 0.846 & 0.78 \\
\hline
\end{tabular}

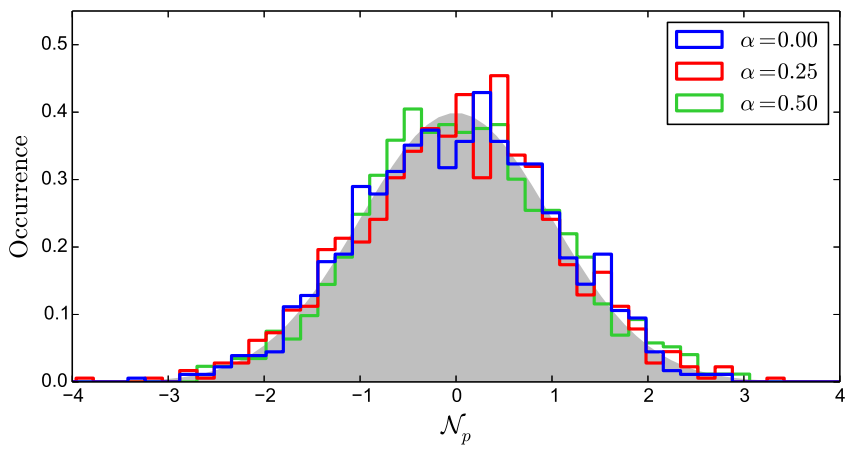

Figure 5. Time-averaging eclipse-depth histogram of the relativeaccuracy statistic for the long-duration (20 times the eclipse duration) synthetic-data simulations. The color code denotes the sample (see legend). The background gray contour denotes a standard normal distribution for comparison. All histograms are normalized such that the integral of each curve adds to one.

ing $\sim 20$ times the eclipse duration (akin to a phase-curve observation). We generate the light curve with the same eclipse configuration and system flux as in Section 4.2, keeping the cadence $\left(17,000\right.$ data points total) and the value of $\sigma_{\omega}$ at 64.5 counts. To conserve the noise rms ratios at $\alpha=0.25$ and 0.5 , we set $\sigma_{r}=774$ and 1549 counts, respectively.

In this case, we find that the time-averaging $\beta$ scaling factors accurately inflate the data uncertainties to account for the time-correlated noise (Fig. 5).

\subsection{Simulation with Spitzer-IRAC Noise}

In this section we describe tests of the correlated-noise estimators for real exoplanet signals from the Spitzer IRAC instrument, which is more compelling than the previous test with synthetic data. We select two published phase-curve observations that are affected by correlated noise, a $4.5 \mu \mathrm{m}$ HD 209458 b (Zellem et al. 2014) and a $3.6 \mu \mathrm{m}$ WASP-14 b (Wong et al. 2015) dataset. The strength of the correlated noise in these two datasets is markedly different. The WASP$14 \mathrm{~b}$ not only presents higher levels of correlated noise (as reported by the time-averaging curves), but also presents sporadic short-duration flux anomalies (dips) along the observation. Thus, these two datasets allow us to test the correlated- 
noise estimators under true instrumental noise as detected by the telescope, under two different correlated-noise regimes. We specifically selected phase-curve observations to remove the astrophysical signals and trace the telescope systematics to the best of our knowledge.

We processed the Spitzer BCD data to obtain raw light curves using the Photometry for Orbits, Eclipses, and Transits (POET) pipeline (Stevenson et al. 2010, 2012a,b, Campo et al. 2011, Nymeyer et al. 2011, Cubillos et al. 2013, 2014). The POET pipeline involves bad-pixel masking (sigma rejection), 2-dimensional Gaussian fitting to determine the target location, and interpolated aperture photometry to obtain raw light curves (for details see, e.g., Cubillos et al. 2014). We remove the first couple hours of observation to avoid the time-dependent systematic.

We model the light curves using Mandel \& Agol (2002) eclipse and transit models, a BLISS map model (to account for the intrapixel effect, Stevenson et al. 2012a), and and a sinusoidal function (for the phase-curve variation, following Zellem et al. 2014):

$$
F(t)=1+c_{0}+c_{1} \cos (2 \pi t)+c_{2} \sin (2 \pi t),
$$

where $c_{0}, c_{1}$, and $c_{2}$ are the model fitting parameters, and $t$ is the time of the observation (measured in orbital phase). To avoid degeneracy with the other fitting parameters, we constrain $c_{0}$ by requiring $F\left(t_{0}\right)=1$, with $t_{0}$ the eclipse midpoint time. We finally remove all astrophysical variations from the signal by dividing out the sinusoidal model and trimming the HD 209458 b phase curves to the span between the eclipse and transit (38.9 h long) and the WASP-14 b phase curve between the transit and eclipse (22.6 h long). The resulting light curves consist of flat curves containing only the intrapixel systematic variation and noise. These curves are our baseline to create the synthetic transit observations.

To construct the trial samples, we inject an eclipse curve at random uniformly distributed times into the baseline, generating 3000 realizations for each dataset. We adopt eclipse parameters (duration, depth, ingress, and egress) similar to the observed values for each dataset (Zellem et al. 2014, Wong et al. 2015).

We analyze the data and outputs in the same manner as in Section 4.1.1. Our fitting model includes an eclipse and a BLISS-map model. In practical terms, we found that many times the MCMC for the wavelet method failed to converge or failed to fit well the entire light curve. This may be result of the wavelet noise model attempting to overfit the transit curve, or because the wavelet cannot appropriately model correlated-noise structure. Thus, for this analysis we trim the dataset to a window of 2.5 times the eclipse duration, centered at the input midpoint.

Figure 6 shows the resulting $\mathcal{N}_{\mathrm{p}}$ histograms for each method and dataset. The $\mathcal{N}_{\mathrm{p}}$ histograms for the WASP-14 b dataset are noticeably more irregular (non-Gaussian) than the histograms for the HD 2019458b dataset. This may be result of the stronger correlated-noise systematics. This clearly complicates the interpretation of the $\mathcal{N}_{\mathrm{p}}$ statistics. Thus, we rely mostly on the coverage-fraction statistic, which is not affected by these nuances.

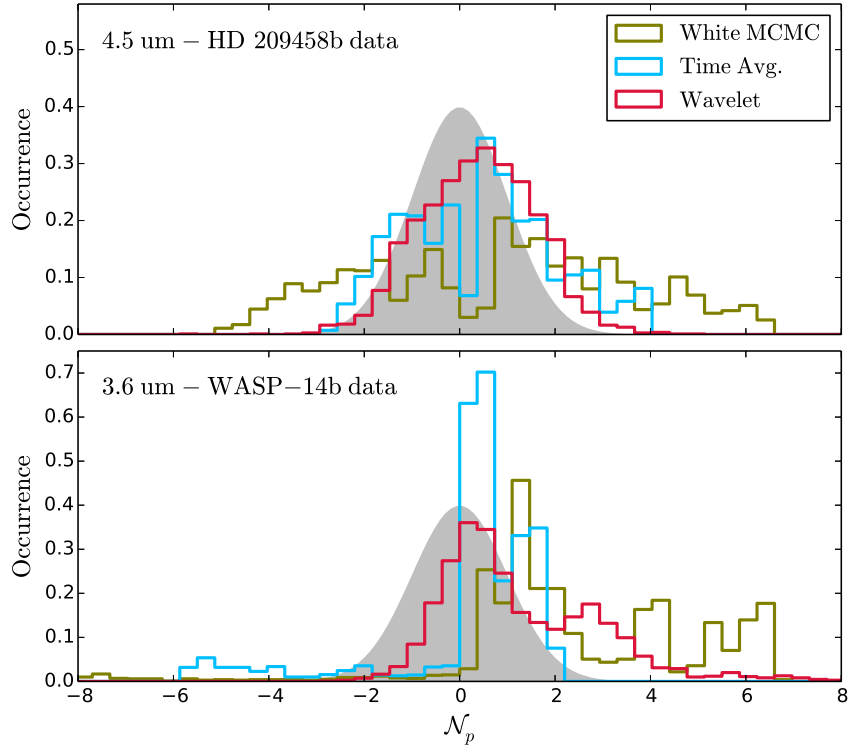

Figure 6. Eclipse-depth histogram of the relative-accuracy statistic for real-noise simulations. The top and bottom panels show the results for the HD $209458 \mathrm{~b}$ and WASP-14 b datasets, respectively. The color code denotes the parameter-estimation method (see legend). The background gray contour denotes a standard normal distribution for comparison. All histograms are normalized such that the integral of each curve adds to one.

Table 3. Relative-accuracy Statistics and Coverage Fraction for Real-noise Simulations

\begin{tabular}{lccc}
\hline \hline Estimation method & $\left\langle\mathcal{N}_{p}\right\rangle$ & $\sigma_{\mathcal{N}}$ & $C_{0.68}$ \\
\hline $\mathbf{4 . 5} \mu \mathbf{m}$ HD 209458 b & & & \\
White MCMC & 0.726 & 2.772 & 0.20 \\
Time Averaging & 0.444 & 1.553 & 0.46 \\
$\quad$ Wavelet & 0.428 & 1.176 & 0.54 \\
3.6 $\boldsymbol{\mu m}$ WASP-14 b & & & \\
White MCMC & 0.720 & 5.901 & 0.15 \\
Time Averaging & 0.202 & 1.682 & 0.64 \\
$\quad$ Wavelet & 1.286 & 1.766 & 0.54 \\
\hline
\end{tabular}

Table 3 presents the $\mathcal{N}_{\mathrm{p}}$ statistic and coverage-fraction results. Again, in both cases, both the time-averaging and wavelet methods improve the parameter estimation with respect to the white analysis (coverage fractions closer to $68 \%$ ). However, none of the analyses completely correct the eclipse depth estimation. The low values of the coverage fractions hint towards an underestimation of the uncertainties, a lack of accuracy, or a combination of both. The irregular shape of the $\mathcal{N}_{\mathrm{p}}$ histograms, particularly for the WASP-14 b dataset, suggests that there are correlations between the accuracy and precision for the trials, which would be expected given the stronger correlated-noise component in the data.

\section{MULTI-CORE MARKOV-CHAIN MONTE CARLO $\left(\mathrm{MC}^{3}\right) \mathrm{CODE}$}

We implemented and made available all of the discussed statistical methods into the open-source Python package Multi-Core Markov-Chain Monte Carlo (MC ${ }^{3}$, https://github.com/pcubillos/MCcubed). Unlike 
other exoplanet model-fitting tools that are tailored to specific tasks, $\mathrm{MC}^{3}$ allows the user to define the modeling function and, thus, it is a general-purpose statistical package. We developed the main bulk of the code in Python, with several extensions written in $\mathrm{C}$, combining simplicity and high performance. The code runs in multiple parallel processors (through the built-in multiprocessing Python package). $\mathrm{MC}^{3}$ provides statistically-robust model optimization (via Levenberg-Marquardt minimization) and credible-region estimation (via MCMC sampling) routines.

The MCMC random sampling is done via the Metropolis Random Walk (MRW, using multivariate Gaussian proposals), the Differential-Evolution Markov-chain Monte Carlo (DEMC, ter Braak 2006), or the Snooker-updater DEMC algorithms (ter Braak \& Vrugt 2008). While the proposal step sizes of the MRW are predetermined by the user and have to be manually adjusted before each run, the DEMC algorithms automatically adjust the scale and orientation of the proposal distribution. To do so, DEMC runs several chains in parallel, computing the proposed jump for a given chain from the difference between the parameter states of two other randomly selected chains. As the chains converge toward the posterior distribution, the proposal jumps will be mainly oriented along the desired distribution and will have adequate scales. Therefore, DEMC improves the MCMC efficiency in two ways: (1) it increases the acceptance rate to optimal levels ( $\gtrsim 25 \%$, Roberts et al. 1997) by better sampling the parameter space, and (2) it eliminates the heuristic need for the user to adjust the proposal jump scales.

The Metropolis-Hastings acceptance rule implements both the ordinary likelihood function (Eq. 3) and the waveletbased likelihood (Eq. 12) using the fourth-order Daubechies wavelet. The priors can be bounded or unbounded uniform, $\log$-scale uniform, or Gaussian. To assess that the MCMC is working properly, the code performs a chain-converge test using the Gelman \& Rubin (1992) statistics. The code also produces several plots to help visualize the results: trace, rms-vs.-bin-size, marginal-posterior, and pairwise-posterior plots can indicate non-convergence, multi-modal posteriors, parameter correlations, correlated noise, or incorrect priors. At the end of the MCMC run the code returns the sampled posterior distribution of the parameters, their best-fitting values, their 68\% HPD credible region, and the acceptance rate of the MCMC. The majority of the routines of this module derive from our POET pipeline and, thus, have been thoroughly tested for years.

The core structure of $\mathrm{MC}^{3}$ consists of a central hub, which drives the MCMC exploration, and the workers, which evaluate the model for the given free parameters. The hub and the worker processes are connected through shared memory. $\mathrm{MC}^{3}$ assigns one $\mathrm{CPU}$ to each worker (i.e., one for each chain). Each cycle (iteration) of the MCMC comprises the following steps: (1) generate the proposal state (the set of free parameters) for each chain, (2) evaluate the model for the proposed state, and (3) compute the Metropolis ratio and accept/reject the proposal state for each chain.

The $\mathrm{MC}^{3}$ code runs from both the shell prompt and the Python interactive interpreter, and is available for Python
2.7 and Python 3. The user can configure the MCMC run either through a configuration file, command line arguments (prompt), and/or function arguments (Python interpreter). The minimum required inputs are the modeling function, the data being fitted, and starting estimate values for the free parameters. As optional arguments, the user can supply the data uncertainties, priors, and any extra arguments of the modeling function (in a manner much like the scipy.optimize.leastq routine). Additionally, the package allows the user to configure multiple features of the MCMC, e.g.: number of chains, number of iterations, burnin length, thinning factor, etc. The repository of the code includes a user manual and guided examples.

\section{CONCLUSIONS}

Time-correlated noise is an important source of uncertainty for faint signals such as exoplanet light curves. Unless all systematics of the data are well understood, the correlated noise must be taken into account to obtain a reliable parameter estimation. We have reviewed three of the most widely used methods to assess time-correlated noise in exoplanet time-series data: time averaging, residual permutation, and wavelet-based likelihood, expanding the limited literature of tests to assess the quantitative results of these techniques. We focused specifically on the case of Spitzer secondary-eclipse time-series data.

We characterized the behavior of the time-averaging $\beta$ correction factor at large bin sizes (the typical case for a transit observation). In this regime the assumed uncertainty of the rms curve is no longer valid, since the posterior adopts the form of a skewed inverse-gamma distribution. We also found the residual-permutation method unsound as a tool for quantifying uncertainty in parameter estimates, because it does not produce ensembles that mimic the behavior of independent draws from a probability distribution. The method is not supported by a consistent statistical basis. Finally, for the wavelet-likelihood method we detected and corrected errors in the published equations Carter \& Winn (2009) and code (Appendix D).

To quantitatively test the performance of these methods, we carried out injection-retrieval simulations on synthetic eclipse light curves, creating a large number of trials for each simulation. We analyzed the results by (1) comparing the expectation and standard deviation of the relative accuracy against a normal distribution (following Carter \& Winn 2009 ) and (2) computing the $68 \%$ coverage fraction (the fraction of trials where the $68 \%$ credible region contained the injected parameter). A correct Bayesian calculation would guarantee matching of the credible region probability and the average coverage (over the parameter space). Note that our simulations used a single true parameter value. A precise test of average coverage would require a substantial amount of computing time. Thus, our conditional coverage tests would indicate a problem with a method only if the coverage differed substantially from the credible level.

In our first simulation, we tested the case when the timecorrelated noise has a power spectral density of the form $1 / f$. Both the time-averaging and the wavelet-likelihood methods 
improved the eclipse-depth estimations over a white MCMC analysis. In this simulation the wavelet analysis is expected to perform well, since the wavelet precisely assumes a noise component with a $1 / f$ power spectral density. We found small differences between the estimated conditional coverage and the credible levels. These diffrences are consistent with expectations, given both the limited precision from the size of the simulations, and the conditional nature of the tests. We also note that the performance of the time-averaging correction can be further improved if the total observation time is much longer than the eclipse duration (as in a phase-curve observation). This arises from the lower signal to noise of the $\mathrm{rms}_{N}$ curve at large bin sizes.

In a further simulation we generated eclipse light-curve samples by injecting an eclipse signal into real Spitzer $3.6 \mu \mathrm{m}$ and $4.5 \mu \mathrm{m}$ IRAC time-series datasets, two sets with notoriously different correlated-noise signals. This experiment allowed us to assess the performance of the timecorrelated estimators without assuming a specific shape of the time-correlated signal. Both the time-averaging and the wavelet-likelihood methods significantly improved the uncertainty estimations compared to a white MCMC analysis, raising the coverage fraction from $15 \%-20 \%$ to $\sim 45-65 \%$. However, they are not perfect, as the coverage fractions are still lower than the expected $68 \%$, suggesting a lack of accuracy and (or) underestimated uncertainties.

In conclusion, it is always better to try to determine the best possible model for the systematics than simply inflating the parameter uncertainties (as in time averaging). However, these sub-optimal noise estimators are better than ignoring time-correlated noise. Luckily, the continuous development of advanced data analysis techniques like Gaussian Processes, Independent Component Analysis, or kernel regression decorrelation (see e.g., Ingalls et al. 2016) will help to improve the best practices required to extract exoplanet data.

Lastly, we presented the open-source Python package Multi-Core Markov-Chain Monte Carlo, available at https://github.com/pcubillos/MCcubed. $\mathrm{MC}^{3}$ implements all of the statistical routines described in this paper, allowing the user to estimate best-fitting model parameters and their credible region, while letting the user provide the modeling function. By releasing our code to the community, we hope not only to provide access to the routines discussed here, but also to encourage researchers to consider open development and cross-validation of the software tools used in the field.

We thank Rebekah Dawson for useful conversations. We thank the referee for comments that significantly improved the quality of the paper. We thank contributors to AstroPy (Astropy Collaboration et al. 2013), SciPy, Matplotlib (Hunter 2007), the Python Programming Language, and the free and open-source community. PC was partly supported by the Fulbright Program for Foreign Students. JB was partly supported by the NASA Earth and Space Science Fellowship Program, grant NNX12AL83H. Part of this work is based on observations made with the Spitzer Space Telescope, which is operated by the Jet Propulsion Laboratory, California Institute of Technology under a contract with NASA. Support for this work was provided by NASA through an award issued by JPL/Caltech and through the NASA Science Mission Directorate's Astrophysics Data Analysis Program, grant NNX13AF38G, and its Planetary Atmospheres Program, grant NNX12AI69G.

\section{Facility: Spitzer(IRAC)}

Software: $\mathrm{MC}^{3}$ (https://github.com/pcubillos/MCcubed), Python

\section{REFERENCES}

Agol, E., Cowan, N. B., Knutson, H. A., Deming, D., Steffen, J. H., Henry, G. W., \& Charbonneau, D. 2010, ApJ, 721, 1861, ADS, 1007.4378 Astropy Collaboration et al. 2013, A\&A, 558, A33, ADS, 1307.6212 Ballard, S. et al. 2010, PASP, 122, 1341, ADS, 1009.0755

Beran, J., Feng, Y., Ghosh, S., \& Kulik, R. 2013, Long-memory processes (Springer, Heidelberg), xviii+884,

ISBN: 978-3-642-35511-0; 978-3-642-35512-7, probabilistic properties and statistical methods

Blecic, J. et al. 2013, ApJ, 779, 5, ADS, 1111.2363

Bouchy, F., Pont, F., Melo, C., Santos, N. C., Mayor, M., Queloz, D., \&

Udry, S. 2005, A\&A, 431, 1105, ADS, arXiv:astro-ph/0410346

Campo, C. J. et al. 2011, ApJ, 727, 125, ADS, arXiv:1003.2763

Carter, J. A., \& Winn, J. N. 2009, ApJ, 704, 51, ADS, 0909.0747

Charbonneau, D. et al. 2005, ApJ, 626, 523, ADS, arXiv:astro-ph/0503457

Cook, S. R., Gelman, A., \& Rubin, D. B. 2006, Journal of Computational and Graphical Statistics, 15, 675

Cubillos, P., Harrington, J., Madhusudhan, N., Foster, A. S. D., Lust, N. B., Hardy, R. A., \& Bowman, M. O. 2014, ApJ, 797, 42, ADS, 1411.3093

Cubillos, P. et al. 2013, ApJ, 768, 42, ADS, 1303.5468

Daubechies, I. 1988, Communications on Pure and Applied Mathematics, 41, 909
Davison, A. C., \& Hinkley, D. V. 1997, Cambridge Series in Statistical and Probabilistic Mathematics, Vol. 1, Bootstrap methods and their application (Cambridge University Press, Cambridge), $x+582$, ISBN: 0-521-57391-2, with 1 IBM-PC floppy disk (3.5 inch; HD) Deming, D. et al. 2014, In prep.

Demory, B.-O., Gillon, M., Seager, S., Benneke, B., Deming, D., \& Jackson, B. 2012, ApJL, 751, L28, ADS, 1205.1766

Deriche, M., \& Tewfik, A. 1993, Trans. Sig. Proc., 41, 2977

Evans, T. M., Aigrain, S., Gibson, N., Barstow, J. K., Amundsen, D. S., Tremblin, P., \& Mourier, P. 2015, MNRAS, 451, 680, ADS, 1504.05942 Fadili, M., \& Bullmore, E. 2002, NeuroImage, 15, 217

Fazio, G. G. et al. 2004, ApJS, 154, 10, ADS

Gelman, A., \& Rubin, D. B. 1992, Statistical Science, 7, 457

Gibson, N. P. 2014, MNRAS, 445, 3401, ADS, 1409.5668

Gibson, N. P., Aigrain, S., Roberts, S., Evans, T. M., Osborne, M., \& Pont, F. 2012, MNRAS, 419, 2683, ADS, 1109.3251

Gillon, M. et al. 2007, A\&A, 471, L51, ADS, arXiv:0707.2261

Gregory, P. 2005, Bayesian Logical Data Analysis for the Physical Sciences (New York, NY, USA: Cambridge University Press), ISBN: 052184150X

Harrington, J., Luszcz, S., Seager, S., Deming, D., \& Richardson, L. J. 2007, Nature, 447, 691, ADS

Hunter, J. D. 2007, Computing In Science \& Engineering, 9, 90

Ingalls, J. G. et al. 2016, ArXiv e-prints, ADS, 1601.05101

Jenkins, J. M., Caldwell, D. A., \& Borucki, W. J. 2002, ApJ, 564, 495, ADS 
Jordán, A. et al. 2013, ApJ, 778, 184, ADS, 1310.6048

Knutson, H. A., Charbonneau, D., Allen, L. E., Burrows, A., \& Megeath, S. T. 2008, ApJ, 673, 526, ADS, arXiv:0709.3984

Knutson, H. A., Charbonneau, D., Cowan, N. B., Fortney, J. J., Showman, A. P., Agol, E., \& Henry, G. W. 2009, ApJ, 703, 769, ADS, arXiv:0908.1977

Lahiri, S. N. 2003, Resampling methods for dependent data, Springer Series in Statistics (Springer-Verlag, New York), xiv+374, ISBN: 0-387-00928-0

Lewis, N. K. et al. 2013, ApJ, 766, 95, ADS, 1302.5084

Mallat, S. 2008, A Wavelet Tour of Signal Processing, Third Edition: The Sparse Way, 3rd edn. (Academic Press), ISBN: 0123743702,9780123743701

Mandel, K., \& Agol, E. 2002, ApJL, 580, L171, ADS, arXiv:astro-ph/0210099

Morello, G. 2015, ApJ, 808, 56, ADS, 1503.05309

Moutou, C., Pont, F., Bouchy, F., \& Mayor, M. 2004, A\&A, 424, L31, ADS, arXiv:astro-ph/0407635

Nymeyer, S. et al. 2011, ApJ, 742, 35, ADS, 1005.1017

Pont, F., Zucker, S., \& Queloz, D. 2006, MNRAS, 373, 231, ADS, arXiv:astro-ph/0608597

Roberts, G., Gelman, A., \& Gilks, W. 1997, Annals of Applied Probability, 7,110

\section{A. BAYESIAN CREDIBLE REGION}

In the Bayesian context, given the posterior probability density, $p(\theta \mid \vec{y})$, of a parameter, $\theta$, given the dataset, $\vec{y}$, the highest posterior density region (or credible region), $R$, is defined by

$$
C=\int_{R} \mathrm{~d} \theta p(\theta \mid \vec{y})
$$

where $\mathrm{C}$ is the probability contained in the credible region. The region $R$ is selected such that the posterior probability of any point inside $R$ is larger than that of any point outside.

In practice, to calculate the credible region, one constructs a histogram of the sampled posterior distribution (normalized such that the sum equals one) and sorts the bins in descending order. Then one sequentially adds the values of $p$ until reaching $C$. The credible-region boundaries are given by the smallest and largest values of $\theta$ for the samples considered in the sum, if the region is contiguous.

\section{B. WLS vs. GLS EXAMPLES}

To gain insight into the difference between weighted least squares estimates (WLS, those considering Eq. (3)) and generalized least squares estimates (GLS, those considering Eq. (4)), we consider examples with a simple correlated noise model: AR(1) autoregressive noise, for regularly sampled data. Our treatment adapts analyses by Zellner (1971) and Sivia \& Skilling (2006) on related models. In this model, the conditional expectation (regression) of the noise for sample $i$ is proportional to the previous noise value; the actual value of the noise is the sum of this expectation and a new innovation contribution, $\nu_{i}$ :

$$
\epsilon_{i}=\phi \epsilon_{i-1}+\nu_{i},
$$

where $\phi$ is the autoregression parameter. The innovations are independent, with zero-mean normal PDFs with standard deviation $s$. The overall model for $f(\theta)$ is a hidden Markov
Ruppert, D., \& Matteson, D. S. 2015, Statistics and data analysis for financial engineering - with R examples, 2nd edn., Springer Texts in Statistics (Springer, New York), xxvi+719, ISBN: 978-1-4939-2613-8; 978-1-4939-2614-5

Sivia, D., \& Skilling, J. 2006, Data Analysis: A Bayesian Tutorial, 2nd edn. (Oxford University Press, USA), ISBN: 0198568320

Southworth, J. 2008, MNRAS, 386, 1644, ADS, 0802.3764

Stevenson, K. et al. 2010, Nature, 464, 1161, ADS, arXiv:1010.4591

Stevenson, K. B. et al. 2012a, ApJ, 754, 136, ADS, 1108.2057

-. 2012b, ApJ, 755, 9, ADS, 1207.4245

ter Braak, C. 2006, Statistics and Computing, 16, 239

ter Braak, C. J. F., \& Vrugt, J. A. 2008, Statistics and Computing, 18, 435

Winn, J. N. et al. 2007, AJ, 134, 1707, ADS, 0707.1908

-. 2008, ApJ, 683, 1076, ADS, 0804.4475

Wong, I. et al. 2015, ApJ, 811, 122, ADS, 1505.03158

Wornell, G., \& Oppenheim, A. 1992a, Signal Processing, IEEE Transactions on, 40, 611

—. 1992b, Information Theory, IEEE Transactions on, 38, 785

Wornell, G. W. 1993, Proceedings of the IEEE, 81, 1428

—. 1996, Signal Processing with Fractals: A Wavelet-based Approach (Upper Saddle River, NJ: Prentice Hall PTR), ISBN: 013120999X

Wu, C.-F. 1981, The Annals of Statistics, 9, 501

Zellem, R. T. et al. 2014, ApJ, 790, 53, ADS, 1405.5923

Zellner, A. 1971, An introduction to Bayesian inference in econometrics (New York: J. Wiley), ISBN: 978-0-471-98165-7

model (HMM): "Markov" indicating that the prediction for the noise at time $t_{i}$ depends only on the noise at the previous time, and not on the whole noise history; and "hidden" because $\epsilon_{i}$ is not directly observed (as it would be in a standard AR(1) model), rather, $y_{i}$ is observed, mixing uncertain model and noise contributions.

The AR(1) model enables recursive construction of the joint distribution for the noise. The model specifies independent normal PDFs for the $\nu_{i}$ terms, so the goal is to express the $\epsilon_{i}$ values entirely in terms of $\nu_{i}$ values. The probability for the first noise sample, $\epsilon_{1}$, is slightly complicated by the fact that it depends on innovations at times before there is data. However, $\epsilon_{i}$ is a linear sum of terms that are each zero-mean normal, so it must itself have a normal PDF, with variance given by the sum of the variances of its contributions. Writing $\epsilon_{i-1}=\nu_{i-1}+\phi_{i-1}$, and recursing, we find

$$
\epsilon_{1}=\sum_{j=0}^{\infty} \phi^{j} \nu_{1-j} .
$$

The standard deviation of each term is $\phi^{j} s$, so the sum of the variances is

$$
\sigma_{\epsilon}^{2}=s^{2} \sum_{j=0}^{\infty} \phi^{2 j}=\frac{s^{2}}{1-\phi^{2}},
$$

provided that $|\phi|<1$. The marginal PDF for $\epsilon_{i}$ at any time is a zero-mean normal with this variance; the noise time series is thus stationary (with the same marginal distribution at each time).

We can write the joint PDF for all noise values in terms of factors that condition on the previous history:

$$
p(\vec{\epsilon})=p\left(\epsilon_{1}\right) p\left(\epsilon_{2} \mid \epsilon_{1}\right) p\left(\epsilon_{3} \mid \epsilon_{1: 2}\right) \cdots p\left(\epsilon_{n} \mid \epsilon_{1: n-1}\right),
$$

with $\epsilon_{i: j}=\left(\epsilon_{i}, \ldots, \epsilon_{j}\right)$. Given the Markov property of the 
AR(1) model, the joint PDF simplifies to

$$
p(\vec{\epsilon})=p\left(\epsilon_{1}\right) \prod_{i=2}^{n} p\left(\epsilon_{i} \mid \epsilon_{i-1}\right) .
$$

Equation (16) implies that $p\left(\epsilon_{i} \mid \epsilon_{i-1}\right)$ is the probability that $\nu_{i}=\epsilon_{i}-\phi \epsilon_{i-1}$. The factors appearing in Eq. (20) are thus

$$
p\left(\epsilon_{1}\right)=\frac{1-\phi^{2}}{s \sqrt{2 \pi}} e^{-\epsilon_{1}^{2} / 2 s^{2}}
$$

and

$$
p\left(\epsilon_{i} \mid \epsilon_{i-1}\right)=\frac{1}{s \sqrt{2 \pi}} \exp \left[-\frac{1}{2 s^{2}}\left(\epsilon_{i}-\phi \epsilon_{i-1}\right)^{2}\right] .
$$

The observation equation, Eq. (1), indicates that the probability for the data, $\vec{y}$, is the probability that the noise values take on the values $\epsilon_{i}=y_{i}-f_{i}(\theta)$. Let $r_{i}(\theta) \equiv y_{i}-f_{i}(\theta)$ denote the residuals from adopting the model with parameters $\theta$. Then the PDF for the data can be written

$$
p(\vec{y} \mid \theta)=\frac{\left(1-\phi^{2}\right)^{1 / 2}}{s^{n}(2 \pi)^{n / 2}} e^{-Q(\theta) / 2 s^{2}},
$$

with

$$
\begin{aligned}
Q(\theta) & =\left(1-\phi^{2}\right) r_{1}^{2}+\sum_{i=2}^{n}\left(r_{i}-\phi r_{i-1}\right)^{2} \\
& =\sum_{i=1}^{n} r_{i}^{2}+\phi^{2} \sum_{i=2}^{n-1} r_{i}^{2}-2 \phi \sum_{i=2}^{n} r_{i} r_{i-1}
\end{aligned}
$$

The first term - the sum of squared residuals-is just the " $\chi$ " " term that appears in WLS (see Eq. (3)). When $\phi \neq$ $0, \operatorname{AR}(1)$ noise correlations introduce new contributions to $Q(\theta)$, including a term resembling a lag-1 autocorrelation. These terms correspond to changes in the model basis projections entailed by the correlations in a GLS analysis, versus a WLS analysis.

\section{B.1. Example: Constant Signal}

The simple case of a constant signal model of unknown amplitude, $f(t ; \mu)=\mu$, is analytically tractable and is illuminating. Substituting $r_{i}=y_{i}-\mu$ and minimizing $Q(\mu)$ leads to the GLS estimate

$$
\hat{\mu}=\frac{w_{n} \bar{y}+w_{2}\left(y_{1}+y_{n}\right) / 2}{w_{n}+w_{2}},
$$

where $\bar{y}$ is the sample mean, $\bar{y} \equiv(1 / n) \sum_{i} y_{i}$, and we have defined weights $w_{n}=n(1-\phi)$ and $w_{2}=2 \phi$. When $\phi=0$ (independent noise), $\hat{\mu}$ is just the sample mean. Otherwise, $\hat{\mu}$ is a weighted average of the full sample mean, and the average of the first and last (i.e., the most widely separated) samples. As $\phi$ approaches unity (strongly positively correlated noise), GLS instructs us to just average the most widely separated samples. In contrast, the WLS estimate is always the full sample mean. The WLS and GLS estimates thus will differ, not just in the uncertainties they assign to the mean, but also in the actual values of the estimates.

$Q(\mu)$ is quadratic in $\mu$, so the likelihood function is a Gaussian function in $\mu$. The reciprocal of the second derivative of

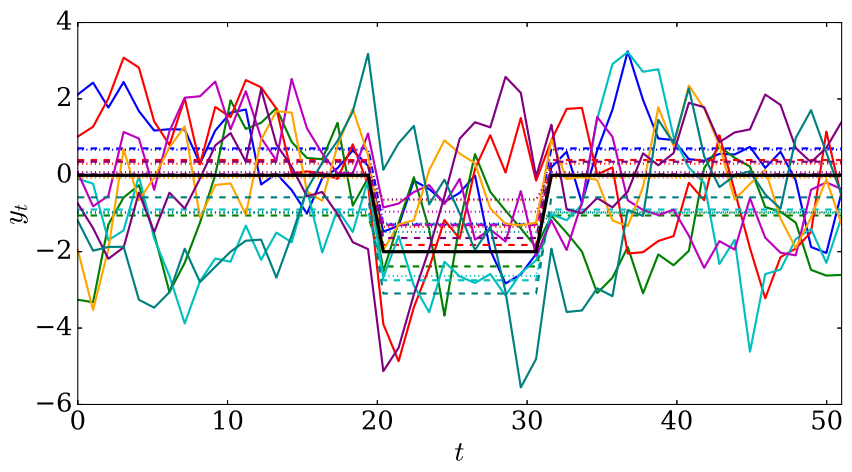

Figure 7. Eight sampled time series from the baseline-dip model with AR(1) noise. Curves of matching color connect the simulated data (solid), the GLS best-fit points (dashed), and the WLS best-fit points (dotted). The solid black curve shows the true (noiseless) function.

$Q(\mu) / s^{2}$ at $\hat{\mu}$ gives the squared standard deviation of this Gaussian,

$$
\sigma_{\mu}^{2}=\frac{s^{2}}{n(1-\phi)^{2}-2 \phi(1-\phi)} .
$$

When $\phi=0$, we have $\sigma_{\mu}=s / \sqrt{n}$, the familiar "root- $n$ " result. As $\phi$ approaches unity, the denominator decreases toward zero, and the uncertainty in $\mu$ grows. Roughly speaking, growing positive correlation decreases the effective sample size, inflating uncertainties. This motivates approaches like time averaging that attempt to account for correlation merely by inflating uncertainties. But such approaches do not account for the effect of correlations on the actual value of a finite-sample estimate.

\section{B.2. Example: Constant Baseline with One Dip}

The effect of correlations on parameter estimates depends on the extent to which the correlations may mimic or distort the projections of the data onto the model components. When a model has components that vary slowly with respect to the correlation scale, the main effect of correlations is to change the effective sample size. But when a model has temporally localized components, correlations can significantly affect, not just the uncertainty scale, but also the best-fit parameter values.

To illustrate this, we used simulated AR(1) noise and the GLS likelihood function to model data generated from a baseline signal of amplitude $a$, with a localized dip of depth $\delta$. We took the dip location and width to be known. For the illustration we report here, we simulated 51 observations with true parameter values $\theta=(a, \delta)=(0,2)$, with the dip spanning 10 samples in the middle of the time series. The noise was generated with an innovation standard deviation $s=1$, and $\phi=0.8$, producing data with autocorrelation time scales $\sim 5$. Figure 7 displays examples of the simulated data and WLS and GLS best-fit function estimates. It is visually apparent that the WLS and GLS estimates sometimes differ.

Figure 8 shows contours of the posterior PDFs for $(a, \delta)$ from a representative simulation where the WLS and GLS estimates differ; here the WLS best-fit estimate is just outside of the $98 \%$ GLS credible region. Even when the WLS and GLS best-fit estimates did not differ too dramatically in, 


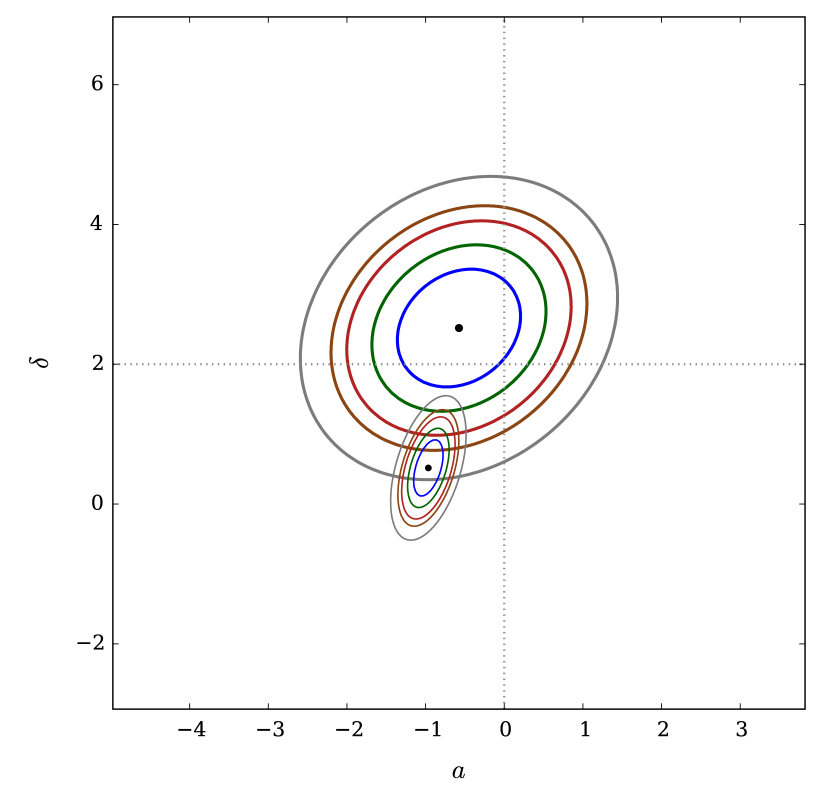

Figure 8. Contours of the posterior PDF for $(a, \delta)$, from GLS (larger, thicker contours) and WLS (smaller, thinner contours) analyses. From inside to outside, the contours bound highest posterior density regions with $50 \%, 75 \%, 90 \%, 95 \%$, and $99 \%$ of the posterior probability. Dots indicate the modes. Crosshairs indicate the true parameter values.

the WLS likelihood function not only has an incorrect uncertainty scale (which one might hope to fix via inflation), but does not correctly capture the shape of the PDF (i.e., the correlation between $a$ and $\delta$ estimates).

The main message of these examples is that noise correlation not only can inflate uncertainties; it can also corrupt parameter estimates, particularly when parameters of interest pertain to temporally localized structure in the model, for which noise correlations can significantly change the data projections needed for accurate inference. Methods that seek to account for correlations only by inflating parameter uncertainties are at best suboptimal (producing larger estimation errors than could be achieved with a good correlated noise model), and can sometimes be significantly misleading.

\section{STANDARD-DEVIATION UNCERTAINTY}

The uncertainty of a parameter estimate in a problem with a fixed model dimension (number of parameters) and growing sample size typically decreases asymptotically at the $\sqrt{M}$ rate. That is, for estimating a Gaussian mean from $M$ samples with the standard deviation $\sigma$, which is known, the uncertainty is $\sigma / \sqrt{M}$. However, this result says nothing about the actual size of the uncertainty at any particular sample size. When $\sigma$ is unknown, it becomes the target of estimation, instead of (or in addition to) the mean. Here, we elaborate on the derivation of the uncertainty for the standard deviation of a Gaussian. The derivation uses the Laplace approximation for a normal standard deviation and its uncertainty, i.e., it finds a Gaussian distribution with a peak and curvature matching the marginal probability density function.

Given a normal distribution of values with unknown mean $\mu$ and standard deviation $\sigma$, let $b_{i}$ be the means for a sample of $M$ groups of samples ("bins") drawn from this distribution. The sample mean, $\bar{b}$, and the sample variance, $s^{2}$, are defined as usual:

$$
\bar{b}=\frac{1}{M} \sum_{i} b_{i}, \quad s^{2}=\frac{1}{M} \sum_{i}\left(b_{i}-\bar{b}\right)^{2} .
$$

If the residual $r_{i}=b_{i}-\bar{b}$ and $r^{2}=\sum_{i} r_{i}^{2}$, then the sample variance becomes $s^{2}=r^{2} / M$.

The likelihood function for our normal distribution with $(\mu, \sigma)$ is:

$$
\begin{aligned}
\mathcal{L}(\mu, \sigma) & =\prod_{i} p\left(b_{i} \mid \mu, \sigma\right) \\
& =\prod_{i} \frac{1}{\sigma \sqrt{2 \pi}} \exp \left[-\frac{\left(b_{i}-\mu\right)^{2}}{2 \sigma^{2}}\right],
\end{aligned}
$$

so the likelihood can be written is terms of $\bar{b}$ and $r$ as:

$$
\mathcal{L}(\mu, \sigma)=\frac{1}{\sigma^{M}(2 \pi)^{M / 2}} \exp \left(-\frac{r^{2}}{2 \sigma^{2}}\right) \exp \left(-\frac{M(\mu-\bar{b})^{2}}{2 \sigma^{2}}(2)\right. \text { ) }
$$

To estimate $\mu$ and $\sigma$, we will adopt a flat prior for $\mu$ and a $\log$-flat prior for $\sigma$, corresponding to $p(\sigma) \propto 1 / \sigma$. Then, the joint posterior probability $p(\mu, \sigma \mid D)$ for $\mu$ and $\sigma$, given the data $D$ is:

$$
p(\mu, \sigma \mid D) \propto p(\sigma) \times \mathcal{L}(\mu, \sigma),
$$

with $p(\sigma)$ the prior probability on $\sigma$.

$$
\left.p(\mu, \sigma \mid D) \propto \frac{1}{\sigma^{M+1}} \exp \left(-\frac{r^{2}}{2 \sigma^{2}}\right) \exp \left(-\frac{M(\mu-\bar{b})^{2}}{2 \sigma^{2}}\right) \beta 1\right)
$$

Calculate the marginal posterior density for $\sigma$ by integrating over $\mu$ :

$$
p(\sigma \mid D) \propto \int \frac{\mathrm{d} \mu}{\sigma^{M+1}} \exp \left(-\frac{r^{2}}{2 \sigma^{2}}\right) \exp \left(-\frac{M(\mu-\bar{b})^{2}}{2 \sigma^{2}}(32)\right.
$$

The $\mu$ dependence is in the last exponential factor, a Gaussian that integrates to $\sigma \sqrt{2 \pi}$. We denote the result as $f(\sigma)$ :

$$
p(\sigma \mid D) \propto \frac{1}{\sigma^{M}} \exp \left(-\frac{r^{2}}{2 \sigma^{2}}\right)=f(\sigma) .
$$

We estimate $\sigma$ with its mode, $\hat{\sigma}$, which maximizes $f(\sigma)$. The first derivative of $f(\sigma)$ is:

$$
f^{\prime}(\sigma)=f(\sigma)\left(\frac{r^{2}}{\sigma^{3}}-\frac{M}{\sigma}\right)
$$

so that setting $f^{\prime}(\hat{\sigma})=0$ gives $\hat{\sigma}=r / \sqrt{M}=s$, as one might expect.

For a simple estimate of the uncertainty, let's consider a Gaussian approximation with mean at $\hat{\sigma}$. The curvature (second derivative) of $\sigma$ at $\hat{\sigma}$ :

$$
f^{\prime \prime}(\sigma)=f^{\prime}(\sigma)\left(\frac{r^{2}}{\sigma^{3}}-\frac{M}{\sigma}\right)+f(\sigma)\left(\frac{M}{\sigma^{2}}-\frac{3 r^{2}}{\sigma^{4}}\right),
$$

determines the standard deviation. When $f(x)$ is of the form of a normal distribution with mean $m$ and standard deviation $w$, it is easy to show that $f^{\prime \prime}(m)=-f(m) / w^{2}$. So, 
if $\delta$ is the standard deviation for $\sigma$, in the normal approximation, that matches the curvature at the peak, we have $\delta^{2} \approx-f(\hat{\sigma}) / f^{\prime \prime}(\hat{\sigma})$. Evaluating Equation (35) at $\hat{\sigma}$, the first term vanishes (since $f^{\prime}(\hat{\sigma})=0$ ), and the remaining term gives an approximate standard deviation of:

$$
\delta \approx \frac{r}{M \sqrt{2}}=\frac{s}{\sqrt{2 M}} .
$$

So, the mean and standard deviation sum for $\sigma$ for large $M$ is:

$$
\sigma=s \pm \frac{s}{\sqrt{2 M}}
$$

\section{WAVELET-LIKELIHOOD ERRATA}

This section reports three erratas found both in the published article of Carter \& Winn (2009) and its associated ASCL code.

First, in the Likelihood equation, Eqs. (32) and (41) of Carter \& Winn (2009), the index for the scale, $m$, should start from 1 instead of 2 . In this case the ASCL code has the correct value.

Next, the variance of the scaling coefficient in the ASCL code for $\gamma=1$, equation (34) of the paper, is missing the factor $2^{-\gamma}=2^{-1}$. The corrected equation should read:

$$
\sigma_{S}^{2}=\frac{\sigma_{r}^{2}}{4 \ln 2}+\sigma_{\omega}^{2}
$$

The expression for the scaling coefficient for $\gamma \neq 1$ also seems to be wrong in the ASCL code. We can compute the variance of the wavelet coefficients following equation (37) of Wornell (1993):

$$
\left\langle\epsilon_{n}^{m} \epsilon_{n}^{m}\right\rangle=\frac{2^{-m}}{2 \pi} \int_{-\infty}^{\infty} \frac{\sigma_{x}^{2}}{|\omega|^{\gamma}}\left|\Psi\left(2^{-m} \omega\right)\right|^{2} \mathrm{~d} \omega .
$$

Assuming an ideal bandpass -i.e., eq. (3) of Wornell (1993) - and with a change of variable, $u=2^{-m} \omega$, we re- produce eq. (24) of Carter \& Winn (2009):

$$
\begin{aligned}
\left\langle\epsilon_{n}^{m} \epsilon_{n}^{m}\right\rangle & =2^{-\gamma m} \frac{1}{2 \pi} \int_{-\infty}^{\infty} \frac{\sigma_{x}^{2}}{|u| \gamma}|\Psi(u)|^{2} \mathrm{~d} u . \\
& =2^{-\gamma m} \frac{2}{2 \pi} \int_{\pi}^{2 \pi} \frac{\sigma_{x}^{2}}{u^{\gamma}} \mathrm{d} u . \\
& =2^{-\gamma m} \frac{\sigma_{x}^{2}}{\pi^{\gamma}} \frac{\left[2^{1-\gamma}-1\right]}{1-\gamma} \equiv 2^{-\gamma m} \sigma_{r}^{2},
\end{aligned}
$$

Analogously, the variance for the scaling coefficient assuming an ideal bandpass -i.e., eq. (10) of Wornell (1993):

$$
\begin{aligned}
\left\langle\bar{\epsilon}_{n}^{m} \bar{\epsilon}_{n}^{m}\right\rangle & =2^{-\gamma m} \frac{1}{2 \pi} \int_{-\infty}^{\infty} \frac{\sigma_{x}^{2}}{|\omega| \gamma}|\Phi(\omega)|^{2} \mathrm{~d} \omega \\
& =2^{-\gamma m} \frac{2}{2 \pi} \int_{0}^{\pi} \frac{\sigma_{x}^{2}}{\omega \gamma} \mathrm{d} \omega \\
& =2^{-\gamma m} \frac{\sigma_{x}^{2}}{\pi^{\gamma}} \frac{1}{1-\gamma} \equiv 2^{-\gamma m} \sigma_{r}^{2} \frac{1}{2^{1-\gamma}-1} .
\end{aligned}
$$

This indicates that $g(\gamma)=1 /\left(2^{1-\gamma}-1\right)$ for $\gamma \neq 1$, the inverse of the value given in the ASCL code from Carter \& Winn (2009). The same result can be derived from equations (16) and (17) of Fadili \& Bullmore (2002). These derivations of $\left\langle\epsilon_{n}^{m} \epsilon_{n}^{m}\right\rangle$ and $\left\langle\bar{\epsilon}_{n}^{m} \bar{\epsilon}_{n}^{m}\right\rangle$ are not valid for $\gamma=1$; in fact, Eq. (45) diverges to $+\infty$ from the left and to $-\infty$ from the right as we approach $\gamma=1$. Then, how can one get to $g(\gamma=1)=$ $1 / 2 \ln 2$ ?.

Lastly, Section 4.1 of Carter \& Winn (2009) mentions that they used a dataset of 1024 elements, and that their DWT produced 1023 wavelet coefficients and 1 scaling coefficient (implying $N_{0}=1$ ). This is inconsistent with the wavelet used (a fourth-order Daubechies wavelet), for which $N_{0}=2$. This wavelet's DWT returns 2 scaling coefficients and 1022 wavelet coefficients (for the given dataset). The ASCL code is also suited to perform a likelihood calculation assuming $N_{0}=1$, resulting in each likelihood term having an $m$ value offset by 1 . 